\title{
The Aesthetic and Cultural Pursuits of Patients with Stroke
}

\author{
Clare O'Connell, Aoife Cassidy,, \\ Desmond O’Neill, MA, MD, FRCPI, AGSF, FRCP (Glasg), ${ }^{*}$ and Hilary Moss, MBA, DipMTh $\ddagger$
}

\begin{abstract}
Background: There has been an increasing interest in the arts in health care, with a suggestion that the arts and aesthetics can augment patient outcomes in stroke and other illnesses. Designing such programmes requires better knowledge of the artistic, aesthetic, and cultural pursuits of people affected by stroke. The aim of this study was to obtain the insights of this group about the profile of art and aesthetic activities in their lives and the influence of stroke on these aspects.
\end{abstract}

Methods:Patients attending a stroke service were administered questions adapted from the Irish Arts Council's 2006 questionnaire on participation in aesthetics and cultural pursuits. Information was also collected on stroke type and present functional and cognitive status. Thirty-eight patients were interviewed. Of these, 20 were inpatients in hospital at the time of the interview and 18 were interviewed in an outpatient setting.

Results: Popular activities included mainstream cinema, listening to music, dancing, attending plays or musicals, and being outdoors. Many patients ceased these activities after their stroke, mostly because of health issues and inaccessibility. Most of the patients valued the importance of the arts in the health-care setting.

Conclusions: This study gives a perspective for the first time on the aesthetic and cultural pursuits of stroke patients before their stroke. It portrays a wide variety of cultural and leisure activities and the cessation of these poststroke. It revealed the restrictions patients felt on gaining access to leisure pursuits both while in hospital and following discharge.

Key Words, Stroke; culture; leisure; aesthetics; arts

\section{Introduction}

There is an emerging interest in the role of arts and aesthetics in the augmentation of the experience of health care. Särkämö et $\mathrm{al}^{1,2}$ demonstrated that patients who listened to their own music or audiobooks for an hour a day (minimum) during their recovery from stroke

From the *Centre for Ageing, Neurosciences and the Humanities, Trinity Centre for Health Sciences, Tallaght Hospital, Dublin; +School of Medicine, Trinity College, Dublin; and $\ddagger$ National Centre for Arts and Health, Tallaght Hospital, Dublin, Ireland.

Received November 27, 2012; revision received April 8, 2013; accepted April 17, 2013.

Patient consent/ethical approval: Ethical approval was granted for this study.

Disclosure: None.

Address correspondence to Hilary Moss, MBA, DipMTh, Tallaght Hospital, Trinity Centre for Health Sciences, Dublin, Ireland. E-mail: mosshi@tcd.ie. showed improvements in sensory processing and verbal memory, compared with a control group. This study illustrates that the mere act of listening to music or audiobooks may help induce long-term plastic changes facilitating improvements in higher cognitive functions and auditory sensory memory or possibly that aesthetic deprivation hinders recovery. ${ }^{3,4}$ Building further on this research, the design of aesthetic interventions ideally requires better knowledge of the artistic, aesthetic, and cultural pursuits of people affected by stroke and any such programme would be better informed by obtaining the opinions of this group about the role of the arts and aesthetics in the health-care environment. We could find no research assessing the nature of cultural and aesthetic pursuits among patients with stroke and thus designed this study.

There is a paucity of literature on the possible benefits of the arts (as opposed to formal art or music therapy) in stroke rehabilitation and the possible hindrance to recovery caused by aesthetic deprivation. Some of the literature quoted as discussing leisure and recreation does not 
actually examine these aspects, ${ }^{5}$ and others dismiss time spent at leisure and recreation as "nontherapeutic." Even in a review of social activities after stroke, some of the articles included in terms of examining leisure and recreation contained no mention of this aspect of recovery. ${ }^{8}$ One insightful article reported a significant curtailment of recreational activities after stroke, 38\% compared with $8 \%$ in age-matched controls. ${ }^{9}$

The existing literature also has a focus on clinical benefits of art therapies and arts and health programmes (particularly music therapy) in areas such as depression, improving communication for patients with aphasia, and as a rehabilitation tool. ${ }^{10,11-16}$ Although there have been many articles written on the benefits of arts in hospital environments ${ }^{1,17-19}$ and particularly arts for older people in various settings, ${ }^{20,21-23}$ only 2 articles focus specifically on the experience of stroke patients in arts poststroke, ${ }^{11,24}$ but none examine stroke patients' interests in arts and culture prestroke and during hospital stay. Three articles were found regarding leisure activities and stroke but not art or cultural interests. $^{25-27}$ If arts and aesthetics play a potentially important role in stroke rehabilitation, more knowledge is needed on the role played by arts, culture, and aesthetics in patients' lives. The study, therefore, also explored the perception of the participants about art activities in hospital and their relevance.

\section{Materials and Methods}

Thirty-eight patients completed a questionnaire administered using questions from a major population study of cultural and aesthetic pursuits in the Irish population. The questionnaire was developed from a previously validated survey by the Arts Council of Ireland, which is used to determine public perception and involvement in the arts. ${ }^{28}$ Relevant questions were taken from the Arts Council questionnaire that related directly to attendance at art events, participation in the arts, and receptive engagement in arts as these were relevant to the aesthetic and cultural pursuits of hospital patients with stroke. Questions relating to purchasing art materials and whether people attended rural or urban art centers were not included as these were deemed less relevant to our study. The questionnaire was then adapted to include questions regarding the patients' aesthetic experience of hospital and the experience of patients' pre- and poststroke. The Arts Council questionnaire included demographic questions including income, level of education, occupation, and marital status. Wherever possible, questions were taken directly from the Arts Council questionnaire as this was a previously validated questionnaire concerned with the aesthetic and cultural interests of the general public.

Most of the questions were closed, with yes/no or a grading of answers (eg, "did you attend art events once in the last year, 1-6 times or more than 6 times?"). The patients were asked only one open-ended question about their preferred art and leisure past-times, both before and after their stroke. Please see Appendix 1 for full questionnaire.

A convenience sample was used of 21 consecutive admissions to the Stroke Service and 17 outpatients from 3 consecutive outpatient clinics at a university teaching hospital. Inclusion criteria included patients who have had a stroke as a primary cause of admission to the Stroke Service. Exclusion criteria were severe cognitive impairment, severe aphasia/language impairment sufficient to preclude participation, or patients who were too ill to take part in the study.

Although 38 patients in total were surveyed, 21 of these were inpatients and 17 outpatients at the time of survey. The full 38 were therefore asked about their art activities prestroke and only the 17 outpatients were asked about participation and attendance at activities poststroke, as clearly the inpatients could not comment on this aspect. However, the full 38 were asked about their engagement in receptive arts poststroke (questions 7 and 9 of the survey), specifically the questions relating to receptive participation in the arts, particularly which media patients used to listen or watch arts and questions relating to reading.

The assessment included demographic variables, the date and type of stroke (ischemic/hemorrhagic), Oxfordshire Community Stroke Project classification, ${ }^{29}$ cognitive screening with the Ottawa 3DY 4-Question Screen for Cognitive Impairment (3DY), ${ }^{30}$ and current functional status via the modified Rankin Scale. ${ }^{31}$ The 3DY cognitive test has 4 questions, giving a maximum score of 4 , a simple way to quickly assess cognitive ability. The modified Rankin Scale is a commonly used scale for measuring the degree of disability or dependence in the daily activities of people who had a stroke or other causes of neurological disability, score runs from 0 to 6 with 0 being no symptoms and 6 being "dead."

\section{Statistical Analysis}

Chi-square tests were undertaken on 7 of the most popular art activities and events. Chi-square tests are used to see if there is a relationship between 2 categorical variables, in this case the group of patients pre-and poststroke. For these statistics, the full 38 patients were counted for reading and listening to music, whereas the 17 poststroke outpatients were used for the other 7 activities.

\section{Results}

\section{Before Stroke}

Twenty-one men and 17 women participated; mean age was 67 years, and their functional and cognitive status is summarized in Table 1. Where possible participants 
Table 1. Patient population

\begin{tabular}{lccccc}
\hline & $\mathrm{N}$ & Age & Men & 3DY & Rankin \\
\hline Inpatients surveyed & 21 & $69( \pm 29)$ & $11(52.4 \%)$ & $2.5( \pm 1.5)^{*}$ & $2.5( \pm 2.5)$ \\
Outpatients & 17 & $64( \pm 24)$ & $10(58.8 \%)$ & $3.7( \pm 1.7)$ & $1.2( \pm 1.8)$ \\
Total & 38 & $67( \pm 27)$ & $21(55.3 \%)$ & $3.1( \pm 2.1)^{*}$ & $2( \pm 3)$ \\
\hline
\end{tabular}

Abbreviation: 3DY, Ottawa 3DY 4-Question Screen for Cognitive Impairment.

*Two participants excluded from 3DY cognitive test

completed the questionnaire independently, but where assistance was needed (eg, where participants had difficulty writing or reading the questions), the researchers assisted patients by reading out questions or filling in the answers, as directed by the participant.

The participants described a wide range of arts and cultural activity. The most popular activity was dancing, with $19(50 \%)$ of patients having participated at some point before their stroke. Some enjoyed this activity right up to the time of their stroke; others danced earlier in life but remembered enjoying it. Popular types of dance were traditional Irish, ballroom, and "60's" dancing.

Other very popular activities were singsongs in pubs, gardening, and "being outdoors." Eight (21.1\%) patients played a musical instrument. Other activities mentioned by patients were "doing impersonations," painting, swimming, going to the gym, and choir singing.

Of the subjects who listened to music regularly, the media used to do so was radio or CDs in most of the cases, with some participants using a digital music player. The popular genres of music within this patient population were classical, country and western, and Irish ballad singers; however, rock, house, and pop music also featured. The preferred genres of music concerts attended were classical, rock, pop, and traditional music.

Popular choices of reading material were true crime, biographies, and the newspaper. Popular television and DVD viewing was sport, murder mysteries, and "oldfashioned" films.

Twenty-seven patients (71.1\%) said they had stopped to look at artwork in a public place; if the patients who said they had noticed such artwork are included, this figure rises to $34(89.5 \%)$.

\section{After Stroke}

For participants who had returned to the community poststroke ( $n=17,44.7 \%)$, there was generally a decline in attendance at art events after stroke. Most stated that the decline in attendance was not because of reasons related to their stroke but because of a variety of other factors including other health issues, "having no one to go with" and inaccessibility of certain art events (local cinema closing down was one example). However, 4 ( $n=4$, $24 \%$ ) patients stated they had simply "lost interest" in attending these events.
The most popular art events prestroke were attending films ( $n=21,55 \%)$, concerts or other musical events $(n=20,53 \%)$, and plays or shows $(n=18,47 \%)$.

Tables 2-5 give further information regarding patients' attendance at art and cultural events pre- and poststroke, their participation in art activities pre- and poststroke, their key leisure/cultural activities, and their opinions about the arts.

The reasons given for not continuing participation in activities after their stroke were health issues because of their stroke $(n=4)$, health issues unrelated to their stoke $(n=5)$, and transport difficulties $(n=3)$. One patient $(n=1)$ had been attending a ballroom dancing evening weekly up until the age of 70 years, when she began to feel "out of place" as she was the oldest person in attendance at the event.

It is interesting to note that there was a decline in participation in all leisure and art pursuits listed after stroke, from reading to attending art exhibitions.

For the whole sample, receptive arts were popular, with listening to music $(n=35,92 \%)$, watching arts on television ( $n=19,50 \%)$, and reading ( $n=25,66 \%$ ) being popular activities prestroke. Interestingly, these were also some of the most popular art activities poststroke (listening to music, $\mathrm{n}=24,63 \%$, watching arts on television, $\mathrm{n}=13,34 \%$, and reading, $\mathrm{n}=14,37 \%$. Reasons cited for the discontinuation of reading as an activity after stroke included headaches $(n=1)$, poor eyesight $(n=$ $5)$, and lack of concentration $(n=6)$.

Table 2. Participation in art activities prestroke

\begin{tabular}{lc}
\hline & $\begin{array}{c}\text { Before stroke, } \\
\mathrm{n}=38\end{array}$ \\
\hline Dancing & 19 \\
Singing & 9 \\
Musical activities (eg, playing an & 8 \\
$\quad$ instrument) & 1 \\
Drama activities (eg, performing in a & \\
$\quad$ play or musical society) & 0 \\
Events (eg, helping organise an art event) & 3 \\
Art (eg, painting, photography, making & \\
$\quad$ films) & 2 \\
Literature (eg, writing) & \\
\hline
\end{tabular}


Table 3. Attendance at art and cultural events of participants prestroke

\begin{tabular}{lc}
\hline & Before stroke, $\mathrm{n}=38$ \\
\hline Cinema & 21 \\
Plays/shows & 20 \\
Comedy & 5 \\
Concerts/musical performances & 18 \\
Art exhibitions & 11 \\
Readings & 0 \\
Festivals & 3 \\
Dance events & 2 \\
\hline
\end{tabular}

Statistical results of chi-square tests are seen in Tables 4 and 5 for both receptive arts and participative arts activities and events pre- and poststroke. Results of pre- and poststroke levels of engagement in the two receptive arts (listening to music and reading) can be seen to be statistically significant. Results are mixed in the five most popular participative activities; the most probable reason for lack of statistical significance in some categories is the very small number in the study.

\section{While in Hospital}

Twenty patients (52.6\%) had stopped to look at artwork in the hospital; if the patients who had noticed the artwork are included, this figure rises to 24 (63.2\%). However, many of the acute patients had not left the stroke unit at the time of assessment and, therefore, may not have had the chance to notice the artwork distributed around the hospital.

Twenty-five inpatients (65.8\%) said they had difficulty gaining access to either the television or the radio/CD player. Nineteen of these (50\% of all patients) said that they missed the television or radio/CD player while being in hospital. Some patients mentioned that they felt it would disturb other patients to have the radio on or that it was impossible to concentrate because of "people coming in and out of the room all the time." The patients who did not have access to the television and/or music but did not miss it stated that this was because of health problems while in hospital such as headaches or that they had lost interest or the power of concentration.

Most patients spoke positively about the artwork on display in the hospital, and there was a general consensus among most of the patients that artwork improves the hospital environment and provides a positive distraction, whereas some patients even mentioned that a habit of talking walks along the corridors to look at the paintings on the walls.

When asked where patients would allocate spending on the arts in hospital, 13 (34.2\%) said they would spend it on art classes for patients, $12(31.6 \%)$ would spend it on concerts for patients, and $12(31.6 \%)$ would allocate it to exhibiting more artwork throughout the hospital. Other suggestions included cooking workshops, computer skills workshops (to get people "back to work" after stroke), singsongs, and dancing evenings (eg, ballroom, set, and 60s dancing).

\section{Attitudes to Arts and Arts Spending}

Thirty-five (92.1\%) participants thought that the arts have become more available in the past 10 years. Thirty respondents $(78.9 \%)$ believed that as much importance should be given to providing art amenities as providing sports amenities. Thirty-one $(81.6 \%)$ participants agreed that today's arts and artists are as important to our society as the legacy of the arts and artists of the past. Twentyfour $(63.2 \%)$ participants stated that they were interested in the arts. Thirty-eight participants (89.5\%) thought that the arts play a valuable and important role in a modern society such as Ireland. Thirty (78.9\%) participants thought that the arts from different cultures give us an insight into the lives of people from different cultures. Thirty-one respondents ( $81.6 \%$ ) believed that the current level of spending on the arts should be maintained even in times of economic recession; however, only 21 (55.3\%) thought that spending by the Arts Council in hospitals should be increased, if it meant a cut or smaller increase elsewhere in arts spending.

\section{Discussion}

This study gives a new perspective on the aesthetic and cultural pursuits of stroke patients before their stroke. It portrays a wide variety of cultural and leisure activities. Most patients had several past-times, which they enjoyed before their stroke, many involving the arts. The most popular activities were dancing, mainstream cinema, listening to music and radio, being outdoors, and gardening.

Most of these activities could not be accessed while in hospital, with the exception of patients who enjoyed listening to music/radio and had a radio or electronic

Table 4. Receptive arts pre- and poststroke (chi-squared test), total sample

\begin{tabular}{lccc}
\hline & $\begin{array}{c}\text { Before stroke, } \\
\mathrm{n}=38\end{array}$ & $\begin{array}{c}\text { After stroke, } \\
\mathrm{n}=38\end{array}$ & $\begin{array}{c}\text { Pearson chi-square } \\
\text { result, asymp. sig }\end{array}$ \\
\hline Listening to music (CD/DVD/radio) & 35 & 24 & .018 \\
$\quad \begin{array}{l}\text { Reading for pleasure (fiction, poetry, biographies, newspapers, } \\
\quad \text { and magazines) }\end{array}$ & 25 & 14 & .001 \\
\hline
\end{tabular}


Table 5. Participative arts pre-and poststroke (chi-squared test), outpatient sample

\begin{tabular}{lccc}
\hline & $\begin{array}{c}\text { No participating } \\
\text { prestroke, } \mathrm{n}=17\end{array}$ & $\begin{array}{c}\text { No participating } \\
\text { poststroke, } \mathrm{n}=17\end{array}$ \\
\hline Dancing & 11 & 1 & .446 \\
Singing & 5 & 1 & .110 \\
Attending cinemalt, asymp. sig & 10 & .001 \\
Attending concerts/musical performances & 9 & 5 & .012 \\
Attending art exhibitions & 4 & 6 & .002 \\
\hline
\end{tabular}

music device at their bedside (eg, radio or digital music/ cassette player).

These findings create an impetus to ensure that needs of patients in hospital are met and that those who design hospital units for people with stroke, and those who provide the clinical services, plan accordingly. Provision to the patient's own choice of music in a manner sensitive to noise for other patients and staff would seem to be a priority, a finding given extra weight by Särkämö's study showing better cognitive and affective outcome for patients with access to their own choice of music after stroke.

For inpatient services, for example, planning outdoor garden spaces may in part meet some of those needs. ${ }^{32}$ In addition, new technological methods may provide "windows" to the outside world for those who are confined by the physical and cognitive changes of stroke. ${ }^{33}$ Attention to access to radio and television, in a manner sensitive to privacy and noise pollution, is important.

Many patients suggested that having a dancing evening or class for inpatients would be enjoyable and sociable. With some imagination, this sort of program might be implemented and be effective at several levels; a program has been described for dance for patients with chronic heart failure. ${ }^{34}$

This study also raises the issue of the change in people's activities after stroke, a topic that is under-researched. ${ }^{27}$ Many of the outpatients interviewed had given up or lost interest in former activities. The reasons for this varied hugely including lack of local facilities, visual problems after stroke, loss of confidence after stroke, and general health problems. Many of the inpatients stated that they were looking forward to getting back their normal daily activities and hoped they would be able to participate in their former past-times.

There was also a lack of patient awareness of the arts programme at the hospital. Despite this, the vast majority of patients expressed interest in it and would have liked to know more about it (including patients who expressed little/no interest in the arts in general). An even greater majority thought that having an arts program in a hospital was important, with many stating that it would give people something to look forward to and would serve as a positive distraction from immediate health problems.
It is clear that one of the obstacles faced in augmenting an arts programme is improving patient awareness of art activities in the hospital.

Patient views on the arts, and arts in health care, showed considerable diversity and should help to inform the design and implementation of art programmes. Although a number of patients were interested in the arts, a similar number showed little or no interest. Notwithstanding, the vast majority believed the arts play a valuable and important role in society, and most did not think arts spending should be cut during the recession. Most also thought that providing art amenities was as important as the provision of sports amenities. These findings mirror the findings of Staricoff et al. ${ }^{35}$

Although increasing academic focus is directed on the contribution of architecture, design of health-care spaces, and visual and performing arts in the hospital environment to patient well-being, ${ }^{36}$ the end user has rarely been adequately consulted or participated directly in this research. A recent study of qualitative research that engaged patient perceptions of arts in health recovered only 54 relevant studies and a number of these recommended further research, a more rigorous approach to consulting patients and larger sample sizes. ${ }^{37}$ If arts and aesthetics are to provide tools for assisting patients through the journey of illness, and a means of providing intellectual and social stimulation to patients while in hospital, more insight into the relevance and nature of aesthetic and cultural pursuits is needed.

This study advances our knowledge by providing a foundation for what has been identified by several articles as a key issue and how to tailor the environment, aesthetic supports, and art programmes for patients with stroke. In addition, it can also help us to learn what to avoid in such programmes, an ethical imperative in any health-care environment. One commentator has noted that "ethics becomes an imperative consideration when art is displayed to a captive population of vulnerable patients who are stressed, fearful, in pain, and may be unable to choose the art "... "inappropriate art styles and subject matter may sometimes worsen outcome. ${ }^{\prime \prime 38}$ Studies such as this one begin an inquiry as to the basis on which selection of art for patients might be made. 


\section{Limitations}

There are a few limitations to the research in this study. First, this study focuses on a patient population, not only small in number but also (because of the geographical location of the hospital) featured a specific section of society. Ten participants $(26 \%)$ had not continued education after primary education and $8(21.1 \%)$ had not completed their second-level education. Only 10 participants ( $26 \%$ ) had attended third-level education. A large majority of the patients were from a lower socioeconomic bracket.

Another limitation of this study was that over half of the participants were still inpatients of the hospital, and therefore, the findings of events and activities enjoyed by stroke patients after their stroke are limited to those who were outpatients as the patients still on the wards had not yet had the opportunity or were still too unwell to participate in many leisure activities. This decreased the "after-stroke" results to a patient population of just 17 .

Although the study indicates that patients did value visual art in the hospital and had preferences regarding aesthetic input in hospital (eg, providing more concerts, exhibitions, and art classes in hospital), the study did not compare inpatient and outpatient responses to these issues. This would be a useful direction for future research and further exploration of the role of arts in rehabilitation during different stages of recovery. It is not possible to make concrete recommendations as to which arts should be provided in different stages of rehabilitation, but this research points to the importance of consulting patients about their aesthetic, cultural, and leisure interests to programme art interventions appropriately at all stages of health care.

\section{Conclusion}

This study provided an insight into the cultural, leisure, and art pursuits of stroke patients. It demonstrated the wide variety of activities preferred by this particular patient population and highlighted many areas and activities that could be potentially incorporated into the art programmes of stroke units. Furthermore, this study has drawn attention to patients' lack of access to resources such as television, radio, and music players and to the restrictions felt by the patient after stroke in relation to involvement in activities and attendance at art events. This study also suggests that stroke patients would benefit from further awareness of the art programme in hospital and that despite the climate of economic cutbacks, patients value the arts highly in both the community and hospital settings.

Acknowledgment: The authors thank the 38 patients who contributed to this study and the staff of the stroke unit at Tallaght Hospital.
Sources of funding: The Meath Foundation part funded the research of H.M.

\section{References}

1. Särkämö T, Tervaniemi M, Laitinen S, et al. Music listening enhances cognitive recovery and mood after middle cerebral artery stroke. Brain 2008;131:866-876.

2. Särkämö T, Pihko E, Laitinen S, et al. Music and speech listening enhance the recovery of early sensory processing after stroke. J Cogn Neurosci 2009;22:2716-2727.

3. Caspari S, Eriksson K, Naden D. The aesthetic dimension in hospital-an investigation into strategic plans. Int J Nurs Stud 2006;43:851-859.

4. Ulrich R. Effects of interior design on wellness: theory and recent scientific research. J Healthcare Inter Des 1991;3:97-109.

5. De Wit L, Putman K, Dejaeger E, et al. Use of time by stroke patients: a comparison of four European Rehabilitation Centers. Stroke 2005;36:1977-1983.

6. Bernhardt J, Dewey H, Thrift A, et al. Inactive and alone: physical activity within the first 14 days of acute stroke unit care. Stroke 2004;35:1005-1009.

7. Huijben-Schoenmakers M, Gamel C, Hafsteinsdóttir T. Filling up the hours: how do stroke patients on a rehabilitation nursing home spend the day? Clin Rehabil 2009; 23:1145-1150.

8. Daniel K, Wolfe CDA, Busch MA, et al. What are the social consequences of stroke for working-aged adults? A systematic review. Stroke 2009;40:e431-e440.

9. Mayo NE, Wood-Dauphinee S, Cote R, et al. Activity, participation, and quality of life 6 months poststroke. Arch Phys Med Rehabil 2002;83.

10. Adsit $\mathrm{P}$, Lee J. The use of art in stroke group therapy. Rehabil Nurs 1986;11:18-19.

11. Beesley K, White J, Alston M, et al. Art after stroke: the qualitative experience of community dwelling stroke survivors in a group art programme. Disability Rehabil 2011; 33:2346-2355.

12. Dolgan S, Tur B, Dilek L, et al. Single music therapy session reduces anxiety in patients with stroke. J Phys Med Rehabil Sci 2011;14:12-15.

13. Knight A, Wiese N. Therapeutic music and nursing in poststroke rehabilitation. Rehabil Nurs 2011; 36:200-204.

14. Yamaguchi S, Akanuma $K$, Hatayama $Y$, et al. Singing therapy can be effective for a patient with severe nonfluent aphasia. Int J Rehabil Res 2012;35:78-81.

15. Miettinnen S. The usefulness of creative therapies in the treatment of depression in patients with aphasia. WFOT Bull 1995;31:54-58.

16. Schauer M, Mauritz K. Musical motor feedback (MMF) in walking hemiparetic stroke patients: randomized trials of gait improvement. Clin Rehabil 2003;17:713-722.

17. Caspari S, Eriksson K, Naden D. Why not ask the patient? An evaluation of the aesthetic surroundings in hospitals by patients. Qual Manage Health Care 2007; 16:280-292.

18. Lane MR. Spirit body healing-a hermeneutic, phenomenological study examining the lived experience of art and healing. Cancer Nurs 2005;28:285-291.

19. Moss H, Nolan E, O'Neill D. A cure for the soul? The benefit of live music in the general hospital. Irish Med J 2007; 100:636-638.

20. Greaves C, Farbus L. Effects of creative and social activity on the health and well-being of socially isolated older 
people: outcomes from a multi-method observational study. J R Soc Promot Health 2006;126:134-142.

21. Symons J, Clark H, Williams K, et al. Visual art in physical rehabilitation: experiences of people with neurological conditions. Br J Occup Therapy 2011;74:44-52.

22. Wikstrom B-M. Older adults and the arts: the importance of aesthetic forms of expression in later life. J Gerontol Nurs 2004;30:30-36.

23. Cohen G. New theories and research findings on the positive influence of music and art on health with ageing. Arts Health 2009;1:48-63.

24. Baumann M, Peck S, Collins C, et al. The meaning and value of taking part in a person-centred arts programme to hospital-based stroke patients: findings from a qualitative study. Disabil Rehabil 2012;35:244-256.

25. Krefting L, Krefting D. Leisure activities after a stroke: an ethnographic approach. Am J Occup Therapy 1991; 45:429-436.

26. Morgan D, Jongbloed L. Factors influencing leisure activities following a stroke, an exploratory study. CJOT 1990;57:223-229.

27. O'Sullivan C, Chard G. An exploration of participation in leisure activities post-stroke. Austr Occup Therapy 2010; 57:159-166.

28. Lunn P, Kelly E. In the frame or out of the picture? A statistical analysis of public involvement in the arts. Dublin, Ireland: National Economic and Social Forum, 2008.

29. Bamford J, Sanercock P, Dennis M, et al. Classification and natural history of clinically identifiable subtypes of cerebral infarction. The Lancet 1991;337:1521-1527.
30. Molnar F, Wells G, McDowell I. The derivation and validation of the Ottawa 3D and Ottawa 3DY three- and four-question screens for cognitive impairment. Clin Med: Geriatrics 2008;2:1-11.

31. Rankin J. Cerebral vascular accidents in patients over the age of 60. II. Prognosis. Scott Med J 1957;2:200-215.

32. Lawson B. Healing architecture. Arts Health 2003; 2:95-108.

33. Hegarty F, Roche D, McCabe C, et al. Using multimedia technology to help combat the negative effects of protective isolation on patients: the open window project-an engineering challenge. J Vis Commun Med 2009; 32:72-77.

34. Belardinelli R, Lacalaprice F, Ventrella $C$, et al. Waltz dancing in patients with chronic heart failure: new form of exercise training. Circ Heart Fail 2008; 1:107-114.

35. Staricoff R, Duncan J, Wright M, et al. A study of the effects of the visual and performing arts in healthcare. J Healthcare Dev 2001;32:25-28.

36. Gesler W, Bell M, Curtis S, et al. Therapy by design: evaluating the UK hospital building program. Health Place 2004;10:117-128.

37. Moss H, Donnellan C, O'Neill D. A review of qualitative methodologies used to explore patient perceptions of arts and healthcare. Med Hum 2012;38:106-109.

38. Ulrich R. The effects of viewing art on health outcomes. In: Frampton S, Charmel $\mathrm{P}$, eds. Putting patients first: best practices in patient-centred care. San Francisco, CA: Jossey-Bass, 2009:129-149. 